\section{Case Reports in Oncology}

\title{
Usefulness of Percutaneous Transesophageal Gastro-Tubing in Patients Receiving Chemoradiotherapy for Advanced Esophageal Cancer: A Case Report
}

\author{
Atsushi Naganuma ${ }^{a, b} \quad$ Ayaka Kishi $^{b} \quad$ Yusuke Ogawa $^{b} \quad$ Tomohiro Kudo $^{a, b}$ \\ Yoshizumi Kitamoto $^{c}$ Tetsushi Ogawa $^{\text {b,d }}$ Hideto Oishi $^{\mathrm{e}}$ \\ aDepartment of Gastroenterology, National Hospital Organization Takasaki General \\ Medical Center, Takasaki, Japan; 'b Nutrition Support Team, National Hospital Organization \\ Takasaki General Medical Center, Takasaki, Japan; 'Department of Radiation Oncology, \\ National Hospital Organization Takasaki General Medical Center, Takasaki, Japan; \\ dDepartment of Surgery, National Hospital Organization Takasaki General Medical Center, \\ Takasaki, Japan; ${ }^{\text {DDepartment }}$ of Surgery, National Hospital Organization Murayama \\ Medical Center, Musashimurayama, Japan
}

\section{Keywords}

Chemoradiotherapy · Esophageal cancer · Percutaneous endoscopic gastrostomy · Percutaneous transesophageal gastro-tubing $\cdot$ Radiation dermatitis

\begin{abstract}
Percutaneous endoscopic gastrostomy (PEG) is often performed for nutritional management in advanced esophageal cancer. We here report a patient who initially received enteral nutrition via a nasogastric tube and in whom the subsequent use of percutaneous transesophageal gastro-tubing (PTEG) circumvented the need for a gastrostomy. It is believed that PEG is less painful than a nasogastric tube. However, we selected PTEG because a PEG would have been within the planned irradiation field and there was concern about radiation dermatitis. We were
\end{abstract}


able to administer chemoradiotherapy with sufficient nutrition via an enteral feeding tube via esophagostomy. PTEG is a very useful tool in patients at risk of radiation dermatitis of the abdomen.

\section{Introduction}

In patients with advanced esophageal cancer, oral intake is often difficult because of esophageal stenosis or peristalsis; percutaneous endoscopic gastrostomy (PEG) $[1,2]$ is therefore often performed for nutritional management $[3,4]$. The present patient had large lymph node metastases around the gastric cardia; thus, the site of construction of a gastrostomy would have overlapped with the planned irradiation area. In general, physical irritation to the skin in irradiated areas should be avoided because it can exacerbate radiation dermatitis [5].

Here, we report a patient with advanced esophageal cancer in whom a tube was constructed using percutaneous transesophageal gastro-tubing (PTEG) [6, 7], instead of using PEG, because there was concern about radiation dermatitis.

\section{Case Report}

A 71-year-old man was admitted to our hospital for management of advanced esophageal cancer. He was $156 \mathrm{~cm}$ tall and weighed $45.2 \mathrm{~kg}$ (body mass index 18.6). He had no previous illnesses but was a heavy smoker and drinker. He had first noted a feeling of constriction in his esophagus 2 months before presentation and had lost $9 \mathrm{~kg}$ in weight over the previous 6 months. By the time of admission, he was unable to even drink water. Upper gastrointestinal endoscopy, esophagography, and contrast-enhanced computed tomography (CT) resulted in a diagnosis of unresectable advanced esophageal cancer (Fig. 1). Therefore, chemoradiotherapy (CRT) was scheduled.

A nasogastric tube and peripherally inserted central catheter (PICC) port were placed and enteral and parenteral nutrition initiated. PEG was considered because it is considered less painful than a nasogastric tube. However, the radiation therapist pointed out that the region of construction of PEG would have overlapped with the radiation field (Fig. 1), creating the possibility that radiation-induced skin reactions would be exacerbated by placement of a PEG tube. We therefore elected to use a PTEG rather than a PEG as the nutritional route (Fig. 2).

We usually use a PTEG starter kit (Sumitomo Bakelite, Tokyo, Japan), which comprises a rupture-free balloon (RFB) catheter (14 Fr $\times 70 \mathrm{~cm}$, balloon volume $10 \mathrm{~mL}$ ), fluorine resincoated straight guidewire $(0.89 \mathrm{~mm} \times 200 \mathrm{~cm})$ for the RFB catheter, puncture needle $(18 \mathrm{G} \times$ $15 \mathrm{~cm})$, J shaped guidewire $(0.89 \mathrm{~mm} \times 100 \mathrm{~cm}), 8 \mathrm{Fr}$ dilator $(2.7 \mathrm{~mm} \times 25 \mathrm{~cm}), 16 \mathrm{Fr}$ dilator $(5.3 \mathrm{~mm} \times 15 \mathrm{~cm}), 18 \mathrm{Fr}$ peel-away sheath dilator, $18 \mathrm{Fr}$ peel-away sheath $(6.0 \mathrm{~mm} \times 10 \mathrm{~cm})$, button type $15 \mathrm{Fr}$ enteral feeding tube, and a fluorine resin-coated guidewire $(0.89 \mathrm{~mm} \times 100$ $\mathrm{cm}$ ) for the enteral feeding tube [8]. First, a fluorine resin-coated straight guidewire was placed in the esophagus via the left nostril. Subsequently, an RFB catheter was placed in the esophagus via a guidewire and $10 \mathrm{~mL}$ of $60 \%$ meglumine diatrizoate diluted 10 -fold with normal saline injected into the RFB. Cervical ultrasound was performed while gently pulling on the balloon. Ultrasound images confirmed that the RFB was in the cervical esophagus. After selecting a puncture site in the left neck, local anesthesia and a skin incision $(10 \mathrm{~mm})$ were performed, after which, avoiding the thyroid gland and large blood vessels, the RFB was 
punctured with an $18 \mathrm{G}$ puncture needle under ultrasonic guidance, the position of the tip of the needle also being confirmed by fluoroscopy. The inner tube of the puncture needle was removed and the J shaped guidewire placed in the RFB after confirming backflow of the contrast agent. The RFB was then collapsed and advanced deep into the esophagus and the guidewire placed into the esophagus. Next, the puncture route was expanded with a dilator with an $18 \mathrm{Fr}$ peel-away sheath (tip $8 \mathrm{Fr}$; proximal side $18 \mathrm{Fr}$ ). The guidewire and dilator were removed and the sheath left in situ. The button type $15 \mathrm{Fr}$ enteral feeding tube $(45 \mathrm{~cm}$ in length) was successfully placed in the stomach via the sheath beyond the lower esophageal stenosis (Fig. 2).

Because the required intake was secured by administering adequate enteral and parenteral nutrition through the enteral feeding tube via esophagostomy and the PICC port, CRT was successfully completed while maintaining the patient's general condition. Fortunately, this patient achieved a complete response to CRT and regained the ability to eat foods orally, enabling eventual removal of the enteral feeding tube via esophagostomy and the PICC port (Fig. 3).

\section{Discussion}

Patients undergoing CRT often experience acute and/or chronic skin reactions that can compromise their quality of life. Many topical agents and specialized wound dressings are available to facilitate prevention and management of radiation dermatitis; however, it is difficult to avoid it completely. Physical irritation to the skin of the irradiated area should be avoided because it can exacerbate radiation dermatitis [5].

In our patient with unresectable advanced esophageal cancer, the site for a PEG overlapped the proposed radiation area because of the location of his lymph node metastases. Therefore, we chose to use a PTEG rather than a PEG, thus enabling administration of CRT and enteral nutrition safely without risking exacerbation of skin damage in the irradiated area by a PEG. Searches of PubMed and Ichushi, which is a bibliographic database created by the Japan Medical Abstracts Society, revealed no reports on radiation therapy through the skin of a PEG site as of October 2019.

In 2003, Oishi et al. were the first to develop a minimally invasive technique for achieving esophagostomy called PTEG that uses an RFB for enteral nutrition and drainage as well as a PEG [6]. The use of an RFB with PTEG allows the interventional physician to create a nonsurgical esophagostomy, even in patients in whom it would be difficult to construct a PEG (i.e., after total gastrectomy or with massive ascites). As demonstrated by the present patient, CRT may constitute a new indication for PTEG.

PTEG is less painful and more cosmetically acceptable than a nasogastric tube (Fig. 2). In our questionnaire survey, all patients who switched from a nasogastric tube to a PTEG indicated that they preferred a PTEG.

Contraindications common to PEG and PTEG are anticoagulant use, liver cirrhosis and portal hypertension, and esophageal gastric varices. PEG and PTEG can cause uncontrollable bleeding and should not be performed in patients with these conditions. An additional contraindication to PTEG is inability to secure a safe puncture line when puncturing the cervical esophagus under ultrasound guidance because of thyroid enlargement or extensive cervical lymphadenopathy. Recurrent nerve palsy may be present in individuals with hoarseness. When the patient has recurrent nerve palsy contralateral to the puncture site, puncture may result in bilateral recurrent nerve palsy with the attendant risk of difficulty in breathing as a 
result of vocal cord closure. Thus, contralateral recurrent nerve paralysis is a contraindication to PTEG; it is therefore important to carefully check for such paralysis prior to performing PTEG. Because the adult cervical esophagus is located on the dorsal side of the left thyroid gland in $90 \%$ of individuals, the approached to PTEG is usually on the left. Another contraindication to this procedure is the presence of esophageal lesions such as tumors, ulcers, and stenosis because they may exacerbate complications such as bleeding and perforation. In the absence of the above-listed contraindications, PTEG is a safe procedure if performed carefully.

In conclusion, PTEG is a very useful tool in the nutritional management of patients with esophageal cancer undergoing CRT in whom PEG construction is not possible because of an overlap with the radiation field and the attendant risk of exacerbating radiation dermatitis. We here report our experience of implementing PTEG in patient with the proposed new indication of CRT.

\section{Acknowledgment}

We are grateful to members of nutrition support team for helpful discussions.

\section{Statement of Ethics}

The patient has given his written informed consent to publish the case.

\section{Disclosure Statement}

The authors declare that they have no conflicts of interest related to the present study.

\section{Funding Sources}

No funding was received.

\section{Author Contributions}

All the authors contributed for the paper, including writing and revising it. All authors read and approved the final manuscript.

\section{References}

1 Gauderer MW, Ponsky JL, Izant RJ Jr. Gastrostomy without laparotomy: a percutaneous endoscopic technique. J Pediatr Surg. 1980 Dec;15(6):872-5.

2 Ponsky JL, Gauderer MW. Percutaneous endoscopic gastrostomy: a nonoperative technique for feeding gastrostomy. Gastrointest Endosc. 1981 Feb;27(1):9-11.

3 Mahawongkajit P, Techagumpuch A, Limpavitayaporn P, Kanlerd A, Sriussadaporn E, Juntong J, et al. Comparison of Introducer Percutaneous Endoscopic Gastrostomy with Open Gastrostomy in Advanced Esophageal Cancer Patients. Dysphagia. 2019 Apr.

4 Ogino H, Akiho H. Usefulness of percutaneous endoscopic gastrostomy for supportive therapy of advanced aerodigestive cancer. World J Gastrointest Pathophysiol. 2013 Nov;4(4):119-25. 
5 Hegedus F, Mathew LM, Schwartz RA. Radiation dermatitis: an overview. Int J Dermatol. 2017 Sep;56(9):909-14.

6 Oishi H, Shindo H, Shirotani N, Kameoka S. A nonsurgical technique to create an esophagostomy for difficult cases of percutaneous endoscopic gastrostomy. Surg Endosc. 2003 Aug;17(8):1224-7.

7 Mackey R, Chand B, Oishi H, Kameoka S, Ponsky JL. Percutaneous transesophageal gastrostomy tube for decompression of malignant obstruction: report of the first case and our series in the US. J Am Coll Surg. 2005 Nov;201(5):695-700.

8 Aramaki T, Arai Y, Takeuchi Y, Sone M, Sato R, Bekku E, et al. A randomized, controlled trial of the efficacy of percutaneous transesophageal gastro-tubing (PTEG) as palliative care for patients with malignant bowel obstruction: the JIVROSG0805 trial. Support Care Cancer. 2019 Sep. 


\section{Case Reports in Oncology}

\begin{tabular}{l|l}
\hline Case Rep Oncol 2019;12:901-908 \\
\hline DOI: 10.1159/000504569 & $\begin{array}{l}\text { @ 2019 The Author(s). Published by S. Karger AG, Basel } \\
\text { www.karger.com/cro }\end{array}$ \\
\hline
\end{tabular}

Naganuma et al: Usefulness of Percutaneous Transesophageal Gastro-Tubing in Patients Receiving Chemoradiotherapy for Advanced Esophageal Cancer: A Case Report

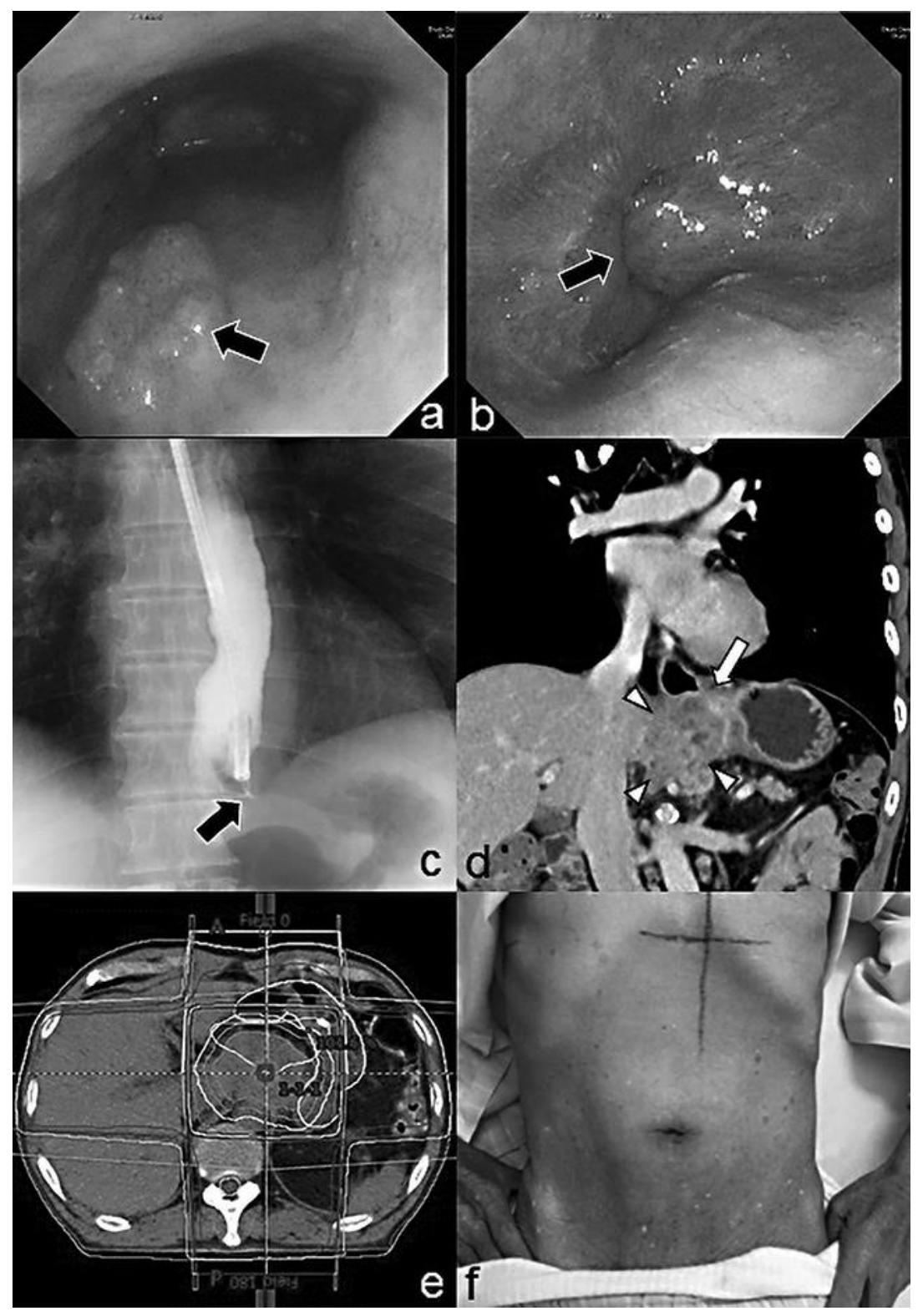

Fig. 1. a Endoscopic image showing primary site of esophageal cancer (squamous cell carcinoma) (black arrow). b Endoscopic image showing esophageal stenosis caused by lymph nodes metastases (black arrow). c Esophageal obstruction as demonstrated by esophageal fluoroscopy (black arrow). $\mathbf{d}$ Esophageal stenosis (white arrow) caused by lymph nodes metastases (white arrow heads) as demonstrated by contrast enhanced computed tomography (coronal image). e Plain computed tomography image for radiation therapy planning. $\mathbf{f}$ Photograph of abdomen showing radiation field marking. 


\section{Case Reports in Oncology}

Naganuma et al:: Usefulness of Percutaneous Transesophageal Gastro-Tubing in Patients Receiving Chemoradiotherapy for Advanced Esophageal Cancer: A Case Report

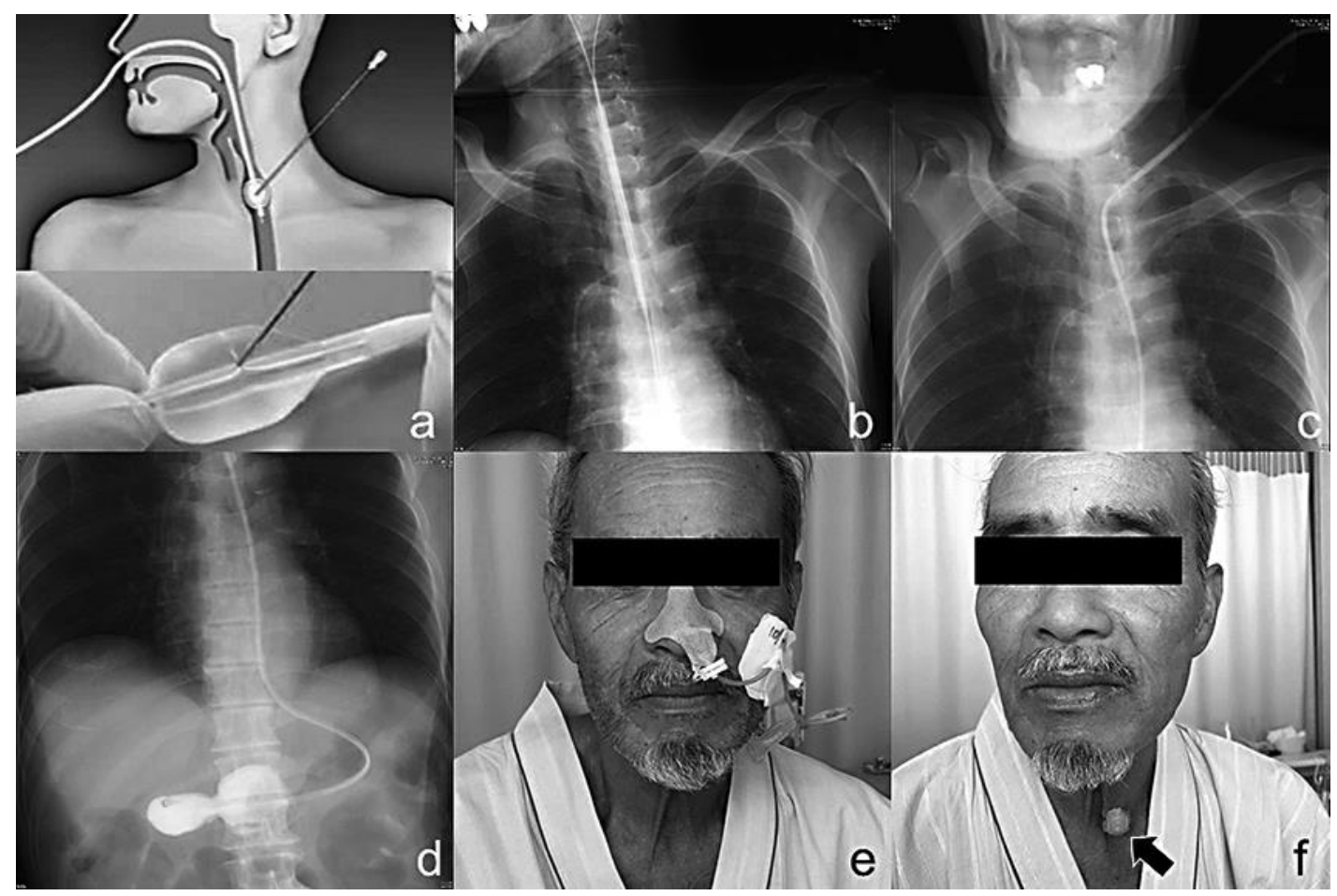

Fig. 2. a Diagrammatic representation showing puncturing of a rupture free balloon (RFB) dilated in the cervical esophagus in an experimental model. $\mathbf{b}$ Plain radiograph showing expansion of the puncture route with a dilator with peel-away sheath (tip $8 \mathrm{Fr}$; proximal side $18 \mathrm{Fr}$ ). c, d Plain radiographs showing a $15 \mathrm{Fr}$ enteral tube placed in the stomach via the cervical esophagus by percutaneous transesophageal gastrotubing (PTEG). e Nasogastric tube in situ. $\mathbf{f}$ A button type 15Fr enteral tube placed in the stomach via the cervical esophagus by PTEG (black arrow) is less painful and more cosmetically acceptable than a nasogastric tube. 


\section{Case Reports in Oncology}

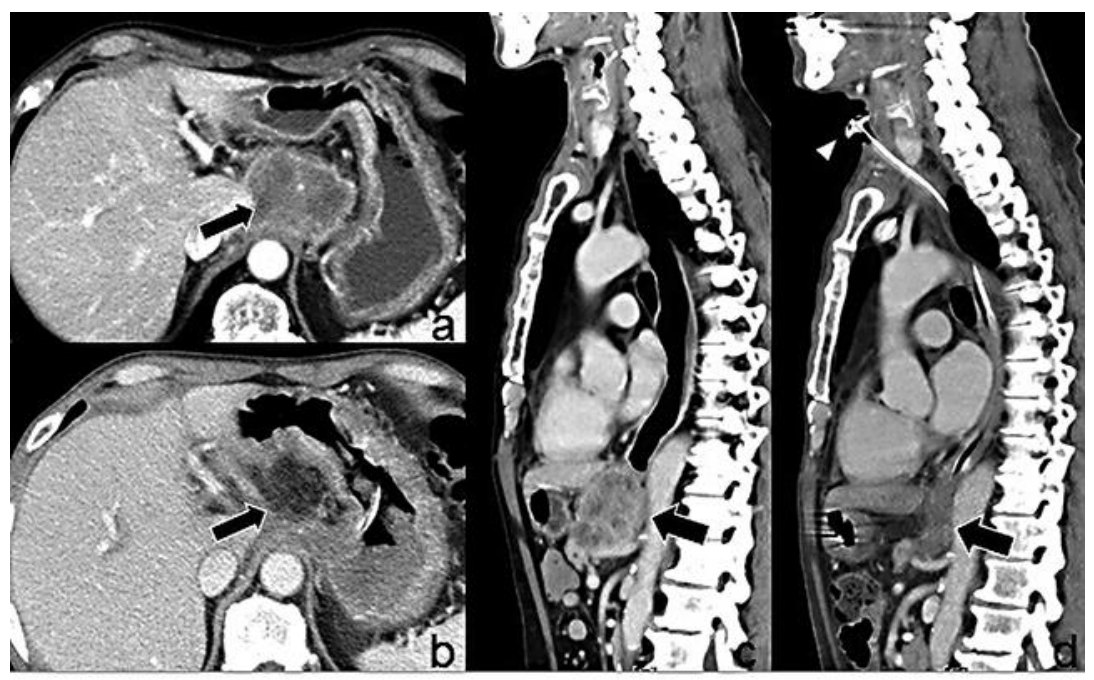

RT

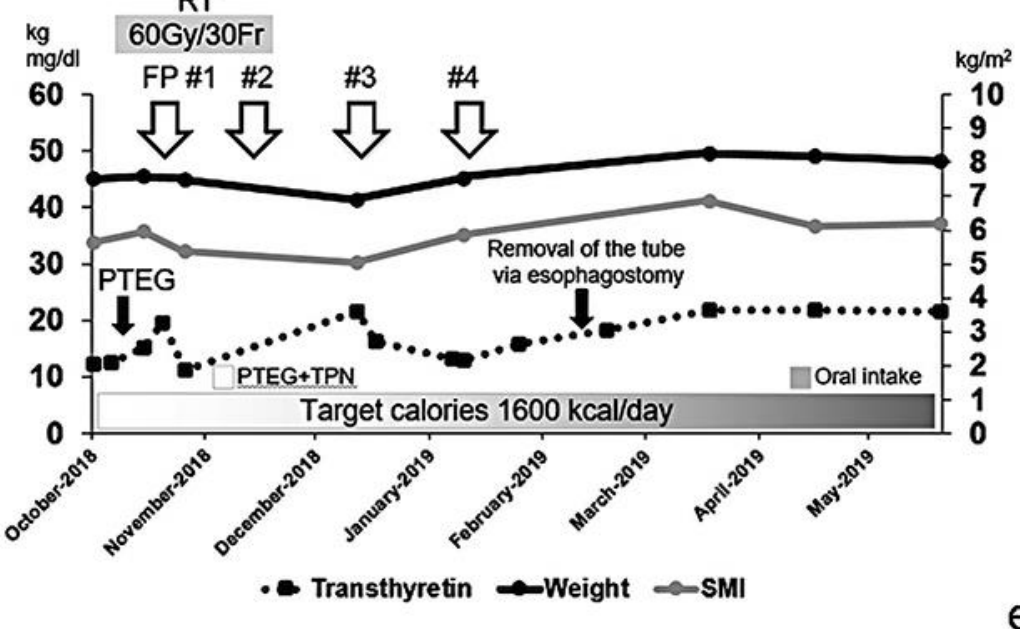

Fig. 3. a Axial contrast-enhanced computed tomography (CT) image before chemoradiotherapy (CRT). Large lymph node metastases are shown by a black arrow. b Axial CT image after CRT. The large lymph node metastases have disappeared (black arrow). c Sagittal CT image before CRT (black arrow). d Sagittal CT image after CRT. The large lymph node metastases have disappeared (black arrow). The white arrow head shows a button type $15 \mathrm{Fr}$ enteral feeding tube. e Clinical course. CRT with 5 -fluorouracil plus cisplatin (FP) was administered. The target daily energy intake of about 1,600 kcal was generally maintained with enteral and parental nutrition. RT; radiation therapy, TPN; total parenteral nutrition, SMI; skeletal muscle index. 